\title{
Traumatic wound rupture after penetrating keratoplasty in Africa
}

\author{
R J C Bowman, D Yorston, T C Aitchison, B McIntyre, C M Kirkness
}

\begin{abstract}
Aim-To investigate risk factors, visual outcome, and graft survival for traumatic wound rupture after penetrating keratoplasty.

Methods-A retrospective analysis of 336 patients who underwent penetrating keratoplasty from 1988 to 1995 .

Results-19 patients (5.7\%) suffered traumatic postoperative wound rupture requiring surgical repair. They were younger (mean age 16.6 years, 95\% CI 13.2-20.6) and more frequently keratoconic $(p=0.01)$ than other patients (mean age 28.9 years, 95\% CI 26.-31.0). Mean postoperative follow up was 37.7 (SD 22.9) months and 24.5 (18.9) months for the rupture and non-rupture patients. Mean interval between keratoplasty and rupture was 18 (21) weeks. The lens was damaged and removed in $37 \%$ of ruptured eyes. For keratoconics, the probability of graft survival at 5 years was lower $(p=0.03)$ in the ruptured eyes $(75 \%)$ than in the nonruptured eyes $(90 \%)$. Endothelial failure was a more common $(p<0.05)$ cause of graft opacification in ruptured grafts than in intact grafts. Of the ruptured eyes, $53 \%$ achieved a final corrected acuity of at least $6 / 18$ and $63 \%$ achieved at least $6 / 60$ compared with $48 \%$ and $71 \%$ of the intact eyes respectively (both $p>0.1$ ). The proportion of keratoconic eyes which achieved at least $6 / 60$ was lower $(p=0.02)$ in the ruptured eyes $(67 \%)$ than the non-ruptured eyes $(87 \%)$. Eyes with wound ruptures of 5 clock hours or greater were less likely $(p<0.05)$ to achieve an acuity of $6 / 18$ and were more likely (p $<0.05$ ) to have an associated lens injury.

Conclusions-Graft rupture is relatively common in African practice, particularly in young keratoconics. Visual outcome and graft survival are not significantly worse than for other grafted eyes, but are significantly worse than for other grafted keratoconic eyes.
\end{abstract}

(Br f Ophthalmol 1999;83:530-534)

Corneal blindness accounts for at least $25 \%$ of blindness in Africa. ${ }^{12}$ The major diseases responsible have been shown to be vitamin $\mathrm{A}$ deficiency, trachoma, and measles. ${ }^{1-3}$ A recent review of penetrating keratoplasty in $\mathrm{Africa}^{4}$ showed good results for keratoconus and corneal dystrophy patients and concluded that corneal grafting in Africa should be used primarily for such patients rather than for the commoner blinding corneal diseases where outcome was less good and for which preventive measures are the key strategy. ${ }^{5}$

One possible and potentially devastating postoperative complication following penetrating keratoplasty is wound rupture secondary to blunt trauma. Comparison of previous reports suggests that this occurs more commonly in African than in Western practice. ${ }^{46}$ Graft survival and visual outcome following traumatic rupture have not previously been reported. This study investigates the incidence, predisposing factors, graft survival, and visual outcomes for this postoperative complication in African patients.

\section{Methods}

A retrospective analysis of patients who underwent penetrating keratoplasty at Kikuyu Eye Unit from 1988 to 1995 was undertaken in July 1996. Any patients no longer attending the clinic were written to and invited to attend for eye examination. Those patients who suffered postoperative wound rupture due to blunt trauma were analysed and compared with those patients whose wounds remained intact, in terms of demographics, diagnosis, graft survival, and visual outcome.

Donor material came mainly from eye banks in the USA and Sri Lanka, preserved in Optisol. The interval between donation and graft ranged from 0 to 21 days (mean 9.7) and donor age ranged between 0.25 and 92 years (mean 61.5). Suture material was 10-0 nylon.

All patients presenting with a ruptured corneal graft wound were repaired as an emergency, usually under general anaesthesia. The area of wound rupture was resutured and reposition of the iris, reformation of the anterior chamber, removal of a damaged lens, and anterior vitrectomy were all performed if necessary.

For assessing individual significance of potential risk factors for postoperative graft rupture, $\chi^{2}$ tests were applied to diagnosis, sex, and suture technique while logistic regression was applied to age. For assessing collective significance of these potential risk factors the simplest model which adequately described the data was found by means of variable selection procedures (including forward stepwise and best subsets) among all possible logistic regression models on these potential risk factors.

Visual outcome was compared between rupture and non-rupture patients, classifying visual acuity according to the World Health Organisation recommended categories of visual loss. ${ }^{7}$ The method of Kaplan and Meier for estimating survival from censored data was 
Table 1 Diagnostic groups for rupture and non-rupture patients

\begin{tabular}{llllll}
\hline & \multicolumn{2}{l}{$\begin{array}{l}\text { Non-rupture } \\
\text { patients }\end{array}$} & & \multicolumn{2}{l}{$\begin{array}{l}\text { Rupture } \\
\text { patients }\end{array}$} \\
\cline { 2 - 3 } \cline { 5 - 6 } Diagnosis & $n$ & $\%$ & & $n$ & $\%$ \\
\hline Keratoconus & 185 & 58.4 & & 18 & 94.7 \\
Corneal scar & 33 & 10.4 & & \\
Corneal dystrophy & 22 & 6.9 & & \\
Bullous keratopathy & 21 & 6.6 & & \\
Herpes simplex keratitis & 20 & 6.3 & & 1 & 5.3 \\
Previous graft failure & 8 & 2.5 & & \\
Bacterial keratitis & 7 & 2.2 & & \\
Trachoma & 7 & 2.2 & & \\
Measles & 4 & 1.3 & & \\
Other & 10 & 3.2 & & \\
Total & 317 & 100 & & 19 & 100 \\
\hline
\end{tabular}

used to estimate the probability of graft survival. The data used for this analysis, for grafts which failed, were the times taken for the graft to become opaque (the end point for failure being the first clinic visit at which the visual acuity had dropped below $6 / 60$ due to corneal oedema). Both the times from the original graft surgery and the time from the repair of the rupture wound were analysed. Grafts lost to follow up were treated as censored data, and survival time was taken to their last clinic attendance, if the graft remained clear. Various log rank tests and proportional hazards models were used to assess relative risks of failure in the rupture and the non-rupture groups, the latter being divided into keratoconus and nonkeratoconus patients.

The possible influence on graft survival and visual outcome of various injury related factors including graft surgery, rupture interval, and others listed in Table 6 was investigated using logistic regression and $\chi^{2}$ tests as appropriate.

\section{Results}

A total of 336 patients underwent penetrating keratoplasty from 1988 to 1995 . Nineteen of these 336 patients $(5.7 \%)$ suffered traumatic postoperative wound rupture requiring surgical repair at some time before July 1996. Typical injuries causing graft rupture in this study were an accidental elbow in the eye from a classmate or a cycling accident. The mean interval between keratoplasty and rupture was 18 weeks (SD 21, range 3-96 weeks). Mean

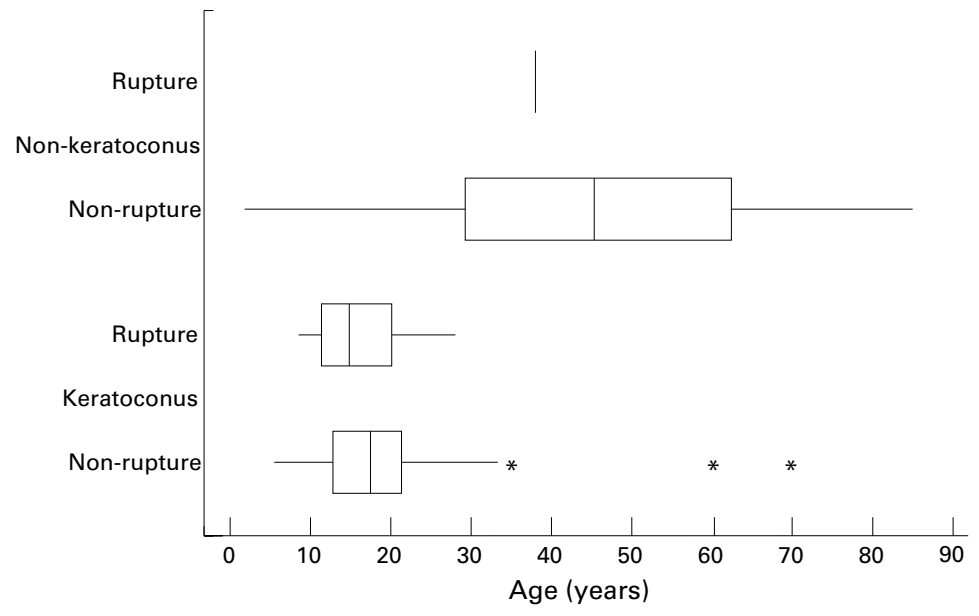

Figure 1 Box plots of age by diagnosis and wound rupture. follow up (from original keratoplasty) for rupture patients was 37.7 (22.9, 1-80) months, significantly longer $(\mathrm{p}<0.05)$ than for the nonrupture patients for whom mean follow up was $24.5(18.9,1-104)$ months. Eighty three patients $(24.7 \%)$ defaulted from follow up including three of the rupture patients. Mean follow up for these 83 patients was 31.6 (22.4, 1-101) months but none of these had attended clinic during the past 12 months. These patients were included in the analyses of survival and visual outcome.

Preoperative diagnoses are shown in Table 1. The non-keratoconus patients were grouped together for the purposes of statistical analysis because none of the nine non-keratoconus diagnoses involved many patients (see Table 1) especially relative to the large number of keratoconus diagnoses (203 patients). Grafts for keratoconus patients (mean $7.6 \mathrm{~mm}$ recipient bed diameter) were larger $(p=0.02)$ than those for non-keratoconus patients (mean 7.2 $\mathrm{mm}$ recipient bed diameter). Corneal vascularisation was less common $\left(\chi^{2}=93.7, p\right.$ $<0.0005)$ in keratoconus patients occurring in five of 203 keratoconic corneas (2\%) compared with 60 of 133 non-keratoconic corneas $(45 \%)$. Within the keratoconus category corneal vascularisation was significantly associated $\left(\chi^{2}=5.4, p=0.02\right)$ with the presence of atopic keratoconjunctivitis, occurring in four of $65(6 \%)$ atopic patients and one of 137 non-atopics (1\%). Eighteen of 203 (8.9\%) keratoconus patients suffered a rupture compared with one of $133(0.8 \%)$ non-keratoconus patients $(\mathrm{p}=0.01)$.

Thirteen of $194(7 \%)$ males in the study suffered a rupture compared with six of $136(4 \%)$ females ( $p>0.3)$. Twelve of $204(6 \%)$ patients with interrupted sutures for their original graft suffered a rupture which was the same rupture rate as for patients who had a continuous suture (seven of 114). Suture data were missing for 18 patients. Suture removal was begun at a mean 7.8 (SD 6.6) months and completed at a mean 12.5 (8.2) months postoperatively with no significant difference between rupture and non-rupture cases ( $p$ $>0.5$ ). In eight of the 19 rupture cases, rupture injury occurred after suture removal was complete and in six of the 19, partial suture removal had been performed. Only two cases of wound rupture occurred within 1 month of an episode of suture removal and there was no significant correlation between the grafttrauma interval and time to initial or final suture removal.

A box plot of age by diagnosis and rupture is shown in Figure 1. The mean age of rupture patients was 16.6 years (95\% CI 13.2-20.6), significantly less $(\mathrm{p}<0.01)$ than that of other patients, 28.9 years (95\% CI 26.7-31.0). Logistic regression analysis confirmed that young age on its own was a significant risk factor for rupture $(\mathrm{p}=0.03)$.

The result of the analysis for assessing collective significance of all potential risk factors was that diagnosis alone contributed all the significant predictive information about rupture. Sex, suture technique, and age added 
Table 2 Postoperative complications occurring after repair of graft rupture

\begin{tabular}{ll}
\hline Complication & Number \\
\hline Endophthalmitis (one with retinal detachment) & 2 \\
Rejection & 2 \\
Bacterial keratitis & 2 \\
Anterior synechiae & 2 \\
Shallow anterior chamber & 1 \\
Herpetic corneal ulcer (HSK original diagnosis) & 1 \\
Corneal haze & 1 \\
Retinal detachment & 1 \\
None & 7 \\
Total & 19 \\
\hline
\end{tabular}

no extra information about the likelihood of rupture. Although correlated with diagnosis, corneal vascularisation, and graft size were not of themselves significant predictors of rupture when all cases were considered. If only keratoconics were considered then graft size and presence of atopy remained insignificant but vascularisation was associated with increased risk $\left(\chi^{2}=6.1, p=0.02\right)$ of wound rupture. Two of five patients with vascularised corneas (40\%) suffered wound rupture compared with 16 of 198 without vascularisation $(8 \%)$.

Postoperative complications which occurred after repair of graft ruptures are listed in Table 2.

The Kaplan-Meier survival curves for keratoconus patients with and without rupture and for non-keratoconus patients without rupture are shown in Figure 2. Since only one non-keratoconus patient had a graft rupture this "group" was excluded from the analysis. Keratoconic eyes without rupture had a better chance $(p=0.03)$ of staying clear (approximately $90 \%$ survival at 5 years) than keratoconic eyes which did suffer a rupture (approximately $75 \%$ survival at 5 years). They also had a better survival chance $(p<0.01)$ than non-keratoconic eyes which did not have a postoperative rupture (approximately 50\% survival at 5 years). There was no statistically significant difference in chance of survival between the keratoconus with rupture and the non-keratoconus without rupture groups ( $p=$ 0.23 ). The significance of these differences was unaltered by whether the survival time for rupture patients was measured from the time of graft surgery or time of rupture repair, because

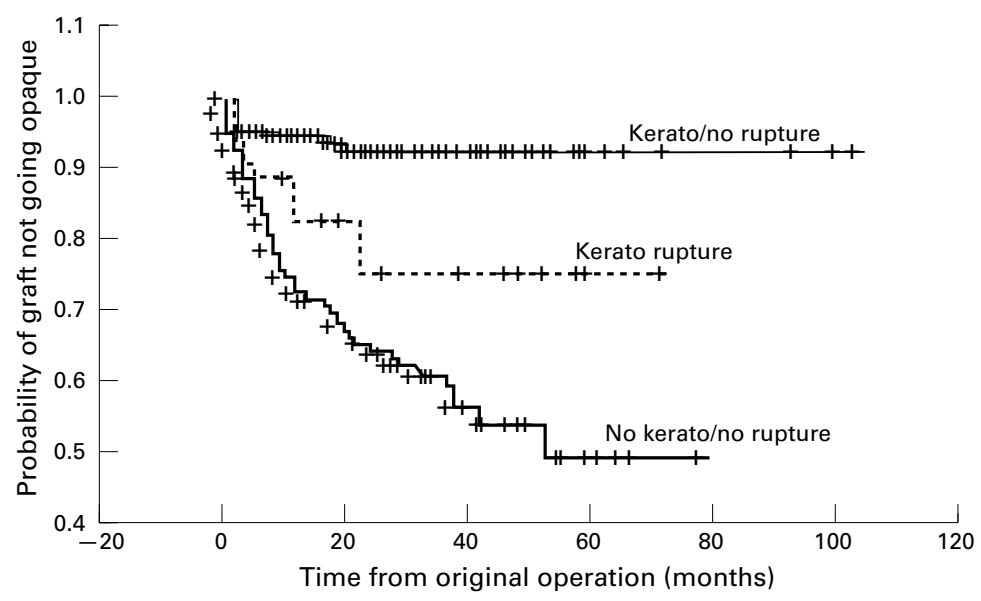

Figure 2 Estimated survival of graft by diagnosis and rupture status.
Table 3 Causes of graft opacity in rupture and non-rupture patients

\begin{tabular}{|c|c|c|c|c|}
\hline \multirow[b]{2}{*}{ Cause of graft opacity } & \multicolumn{2}{|c|}{$\begin{array}{l}\text { Non-rupture } \\
\text { patients }\end{array}$} & \multicolumn{2}{|c|}{$\begin{array}{l}\text { Rupture } \\
\text { patients }\end{array}$} \\
\hline & $n$ & $\%$ & $n$ & $\%$ \\
\hline Bacterial ulcer & 16 & 5.0 & 1 & 5.3 \\
\hline Rejection & 15 & 4.7 & & \\
\hline Endothelial failure & 7 & 2.2 & 3 & 15.8 \\
\hline Recurrent disease & 6 & 1.9 & 1 & 5.3 \\
\hline Persistent epithelial defect & 6 & 1.9 & & \\
\hline Primary graft failure & 5 & 1.6 & & \\
\hline Endophthalmitis & 4 & 1.3 & & \\
\hline Vitreous touch & 1 & 0.3 & & \\
\hline None (graft clear) & 257 & 81.1 & 14 & 73.6 \\
\hline Total & 317 & 100 & 19 & 100 \\
\hline
\end{tabular}

Table 4 Visual results in rupture and non-rupture patients

\begin{tabular}{llllll}
\hline & \multicolumn{2}{l}{$\begin{array}{l}\text { Non-rupture } \\
\text { patients }\end{array}$} & & \multicolumn{2}{l}{ Rupture patients } \\
\cline { 2 - 3 } \cline { 6 - 6 } $\begin{array}{llllll}\text { Latest corrected } \\
\text { visual acuity }\end{array}$ & $n$ & $\%$ & & $n$ & $\%$ \\
\hline$\geqslant 6 / 18$ & 152 & 47.9 & & 10 & 52.6 \\
$<6 / 18-6 / 60$ & 72 & 22.7 & & 2 & 10.5 \\
$<6 / 60-1 / 60$ & 16 & 5.0 & & 0 & 0 \\
$<1 / 60$ & 77 & 24.3 & & 7 & 36.9 \\
Total & 317 & 100 & & 19 & 100 \\
\hline
\end{tabular}

most ruptures occurred a short time after surgery (mean 18 weeks).

The causes of graft failure in the two groups are shown in Table 3. Endothelial failure was a more common cause of opacity in ruptured grafts than intact grafts $(p=0.004)$. There was no significant association between endothelial failure and delayed wound repair in rupture cases.

Visual outcomes for the two groups are shown in Table 4 . Fifty three per cent of the ruptured eyes achieved a final corrected acuity of at least $6 / 18$ and $63 \%$ achieved at least $6 / 60$, not significantly different ( $p>0.1$ ) from $48 \%$ and $71 \%$ of the non-rupture eyes respectively. If the visual results of keratoconus eyes are analysed separately proportions of ruptured $(56 \%)$ and non-ruptured eyes (64\%) which achieved at least $6 / 18$ vision were not significantly different $(p>0.05)$ but a smaller proportion $(\mathrm{p}=0.02)$ of ruptured eyes $(67 \%)$ than non-ruptured eyes $(87 \%)$ achieved at least $6 / 60$.

Causes of poor visual outcome (acuity less than 6/18) in rupture patients are listed in Table 5. Details of the rupture injuries are shown in Table 6 . None of these factors, nor the cause of the blunt injury, had any significant influence on graft survival but eyes with wound ruptures larger than 5 clock hours were more likely $(\mathrm{p}<0.005)$ to have additional

Table 5 Causes for low vision in patients with latest acuity $<6 / 18(n=9)$

\begin{tabular}{ll}
\hline Cause for low VA & $\begin{array}{l}\text { Latest } \\
\text { Snellen } V A\end{array}$ \\
\hline Not refracted & $6 / 24$ \\
Partial vascularisation & $6 / 36$ \\
Uncorrected apkakia & $1 / 60$ \\
Graft failure & $\mathrm{HM}$ \\
Graft failure after disease recurrence & $\mathrm{HM}$ \\
Primary graft failure & $\mathrm{HM}$ \\
Total retinal detachment and graft failure & $\mathrm{PL}$ \\
Endophthalmitis and total retinal detachment & $\mathrm{PL}$ \\
Endophalmitis secondary to bacterial keratitis & \\
$\quad$ requiring evisceration & $\mathrm{NPL}$ \\
\hline
\end{tabular}


Table 6 Injury characteristics of ruptured eyes

\begin{tabular}{llll}
\hline Injury factor & Yes & No & Total \\
\hline Delayed presentation $>24$ hours & 5 & 14 & 19 \\
Size $>$ 5 clock hours & 4 & 15 & 19 \\
Lens injury & 7 & 12 & 19 \\
Vitrectomy required & 6 & 13 & 19 \\
Iris prolapse & 11 & 8 & 19 \\
Flat anterior chamber & 9 & 10 & 19 \\
\hline
\end{tabular}

lens injury and were less likely $(\mathrm{p}<0.05)$ to achieve a visual acuity of $6 / 18$ or better.

The most recent postoperative refractive results were not significantly different ( $p$ $>0.05$ ) between the rupture and non-rupture patients. The mean postoperative cylinders values were 4.2 (SD 5.4) dioptres for rupture patients and 1.5 (18.9) dioptres for the non-rupture patients. The mean spherical equivalent magnitudes were 3.2 and 5.1 dioptres in the rupture and non-rupture patients respectively.

\section{Discussion}

Traumatic wound rupture has been reported as a postoperative complication of penetrating keratoplasty in two previous studies. Kirkness et $a l^{6}$ reported a $2 \%$ rate in keratoconic eyes in the UK and a previous study from our centre in Kenya $^{4}$ (which included some of the patients presented in this series) reported a $5.4 \%$ rate for all graft patients. The rupture rate in this study was $5.7 \%$ overall but $9.5 \%$ in keratoconic eyes. This high rate compared with the UK study reflects the generally higher levels of ocular trauma seen in Africa compared with the West. This results from harsher, more agricultural lifestyles, higher assault rates, and different cultural attitudes. The proportion of ocular injuries which result in wound rupture is impossible to estimate from this study since minor injuries usually go unreported by patients. The high level of trauma in graft patients occurs despite the fact that they are a highly selected, atypical African population. For patients to be offered penetrating keratoplasty, they must live within easy travelling distance of Nairobi, and persuade the surgeons that they have the motivation and the means to comply with meticulous postoperative care of their eyes and to attend for very regular follow up for some years following the surgery. These criteria were demonstrated by the quick presentation of the patients after injury.

The size of the cohort (336 patients) was only large enough to detect risk factors which increased the chance of rupture by about two and a half times at $80 \%$ power and $95 \%$ confidence and minor risk factors may have been missed. Nevertheless, two major individual risk factors for postoperative rupture were identified: young age and keratoconus. These two factors are strongly dependent on each other since keratoconus patients (mean age 17.7 years) were significantly $(p<0.0005)$ younger than non-keratoconus patients (mean age 39.8 years). Multiple analysis of risk factors showed that a diagnosis of keratoconus effectively contained all the predictive information on the possibility of rupture as, in general, younger people had keratoconus.
Whether keratoconus itself is a risk factor for wound rupture is not clear because of its strong association with young age. Although the thickness of the corneal host bed may be reduced, correct suture technique, passing down to the level of Descemet's membrane should not result in a weak wound other authors have not reported high rates of wound rupture in keratoconics. ${ }^{8}$ It is conceivable that corneal vascularisation, more common in nonkeratoconics, contributes to quicker wound healing hence reducing the risk of rupture. Against this, however, was the lack of overall correlation between vascularisation and rupture risk and the increased risk of rupture in keratoconus eyes with vascularisation. Corneal vascularisation in keratoconics may be associated with more severe disease and a thinner and structurally weaker host bed. There was an association between presence of atopic keratoconjunctivitis and vascularisation in keratonics which might suggest that these patients rub their eyes more, possibly weakening the wound. Against the eye rubbing hypothesis, however, was the lack of association between atopic keratoconjunctivitis itself and rupture risk. The host and donor corneal disc diameters were larger in keratoconic patients than non-keratoconic patients which means that the wound was longer but there was no direct association between wound size and risk of rupture.

Keratoconics tend to be grafted at a younger age at this unit than in Western practice (mean age 17.7 years compared with 22.1 in the UK series with $2 \%$ rupture $^{6}$ ) because of the severity of atopic keratitis and keratoconus in the local population. Younger patients tend to lead a more vigorously active lifestyle, many of these patients still having been at school, and therefore are more vulnerable to ocular injury. Young age may therefore contribute to the increased rupture rate though in our series younger keratoconics were not at significantly greater risk of rupture than older keratoconics. Graft sutures are removed earlier in our patients than in Western practice. This is because of a higher rate of suture infection and relatively quick healing of young keratoconics. It is possible that such early suture removal might predispose to wound weakness but this study has not found any evidence to support this, rupture patients not having had their sutures removed significantly earlier than nonrupture patients.

Analysis of all grafted eyes in this series did not reveal any significant reduction in graft survival or visual outcome from the occurrence of rupture. This was partly because 18 of the 19 rupture patients were keratoconic and such patients have been shown to have better graft survival and visual outcome than patients with other diagnoses. ${ }^{49}$ The adverse effect of rupture was therefore masked by the favourable outcome in keratoconic eyes. When the analysis was restricted to keratoconus patients there was a significant reduction in probability of 5 year graft survival and a significant reduction in the proportion of patients achieving a latest corrected visual acuity of at least $6 / 60$. 
Secondary endothelial failure was a more common cause of graft opacity in ruptured eyes than in non-ruptured eyes presumably because of endothelial damage sustained during the blunt injury and the following hours with a flat anterior chamber. Other causes of poor visual outcome included two cases of endophthalmitis. One occurred acutely after repair of the injury and was accompanied by total retinal detachment. The other case occurred 15 months after repair and was caused by late presentation of bacterial keratitis. Another patient suffered total inoperable retinal detachment as a late complication. Kirkness et $a l^{6}$ reported the occurrence of a giant retinal tear in one of four patients (all of whom lost intraocular contents) with graft rupture. In our series the patients with late retinal detachment, acute endophthalmitis and acute graft failure after repair all had wound sizes over 5 clock hours and lens injury. Such wound size was associated with significantly greater likelihood of lens injury and with worse visual outcome. Longer follow up may reveal the onset of further late complications such as glaucoma related to anterior synechiae.

In conclusion, postoperative wound rupture is relatively common after penetrating keratoplasty in Africa and is much more common in keratoconics and more common in younger patients. The lens was lost in $37 \%$ of rupture cases. Wound rupture adversely affected graft survival and visual outcome in keratoconics but despite this, probability of graft survival at 5 years was $75 \%$ and over $50 \%$ of patients had a latest corrected acuity of $6 / 18$ or better. The risk of postoperative wound rupture is not therefore judged to be a reason for discouraging such patients from undergoing penetrating keratoplasty. Instead, we recommend specific advice on protection of the eye to young and keratoconic patients undergoing penetrating keratoplasty. Particular care in the classroom should be emphasised, cycling discouraged, and the provision of protective spectacles to all such patients at time of discharge from hospital is advisable.

The authors wish to thank Dr M Wood for allowing access to his patients and to Christoffel Blindenmission for assistance with funding this work

1 Whitfield R, Schwab L, Ross-Degnan D, et al. Blindness and disease in Kenya: ocular status survey results from the Kenya Rural Blindness Project. $\mathrm{Br} \quad \mathcal{f}$ Ophthalmol 1990;74:333-40.

2 Faal H, Minassian D, Sowa S, et al. National survey of blindness and low vision in the Gambia: results. $\mathrm{Br} F \mathrm{Oph}$ thalmol 1989;73:82-7.

3 Foster A, Yorston D. Corneal ulceration in Tanzanian children: relationship between measles and vitamin A deficiency. Trans Roy Soc Trop Med Hyg 1992;86:454-5.

4 Yorston D, Wood M, Foster A. Penetrating keratoplasty in Yorston D, Wood M, Foster A. Penetrating keratoplasty in
Africa: graft survival and visual outcome. Br f Ophthalmol Africa: graft sur

5 Foster A, Gilbert C. Community efforts in the reduction of corneal blindness in developing countries. Refract Corneal Surg 1991;7:445-8.

6 Kirkness CM, Ficker LA, Steele ADMcG, et al. The success of penetrating keratoplasty for keratoconus. Eye 1990;4: 673-88.

7 World Health Organisation. Strategies for the prevention of blindness in national programmes. Geneva: WHO, 1984.

8 Paglen GP, Fine M, Abbott RL, et al. The prognosis for keratoplasty in keratoconus. Ophthalmology 1982;89:651-

9 Vail A, Bradley BA, Easty DL, et al. Corneal graft survival and visual outcome. A multicentre study. Ophthalmology 1994;101:120-7. 